\title{
Energy Efficient Error Correction in Mobile TV
}

\author{
Xiaoying Shao, Roel Schiphorst, and Cornelis H. Slump \\ University of Twente, Enschede, the Netherlands \\ E-mail: \{x.shao, r.schiphorst and c.h.slump\}@ewi.utwente.nl
}

\begin{abstract}
The current error correction layer of digital mobile TV is designed for worst case scenarios, which often do not apply. In this paper, we propose a new opportunistic error correction layer based on fountain codes and a resolution adaptive ADC, which has been integrated into the OFDM-based physical layer. The key element in the new proposed system is that only packets are processed by the receiver which have encountered highenergy channels. Others are discarded. With this new approach, around $84 \%$ of the energy consumption in ADCs can be saved compared with the conventional mobile TV system under the same channel conditions.
\end{abstract}

Index Terms-mobile TV, fountain codes, resolution adaptive ADCs, OFDM

\section{INTRODUCTION}

Low power consumption in battery-powered digital mobile TV receivers is a highly desirable feature. Consumers expect their devices to operate for several hours on a single battery charge. In wireless LAN systems, the Analog-to-Digital Converter (ADC) can consume up to $50 \%$ of the total baseband power budget [1]. Similar numbers are expected for mobile TV systems, as both systems are based on Orthogonal Frequency Division Multiplexing (OFDM). In this paper, we propose a novel scheme to reduce the power consumption of the ADC by combining a resolution adaptive ADC architecture with opportunistic error correction.

The mobile wireless channel is a hostile environment. It suffers from time-varying multi-path propagation and high levels of man-made interference, such as from GSM and $3 \mathrm{G}$ wireless systems [2]. In effect, mobile TV channels are modeled as time-varying frequency selective channels. Because of the frequency-selective fading and the interference, the worst-case dynamic range of the received signal is very high, typically around $52 \mathrm{~dB}$ (TU6 channel ${ }^{1}$ ) in addition to the range needed for proper signal detection (SNR). Given a traditional fixed-resolution ADC, this high dynamic range leads to a high number of bits and therefore to high power consumption [3].

However, the worst-case conditions do not always apply. Therefore, we use a resolution adaptive ADC which selects the minimum number of bits according to the current channel. As a result, the power consumption of the ADC is reduced under most, i.e. non worst-case, channel conditions.

A further resolution reduction of the ADC can be achieved by using a novel opportunistic error correction scheme that integrates into the physical layer. This approach allows us to discard some part of the channel with deep fading. Take Figure 1 as an example, the dynamic range of the whole channel is

\footnotetext{
${ }^{1}$ TU6 channel: the Typical Urban 6-path channel model.
}

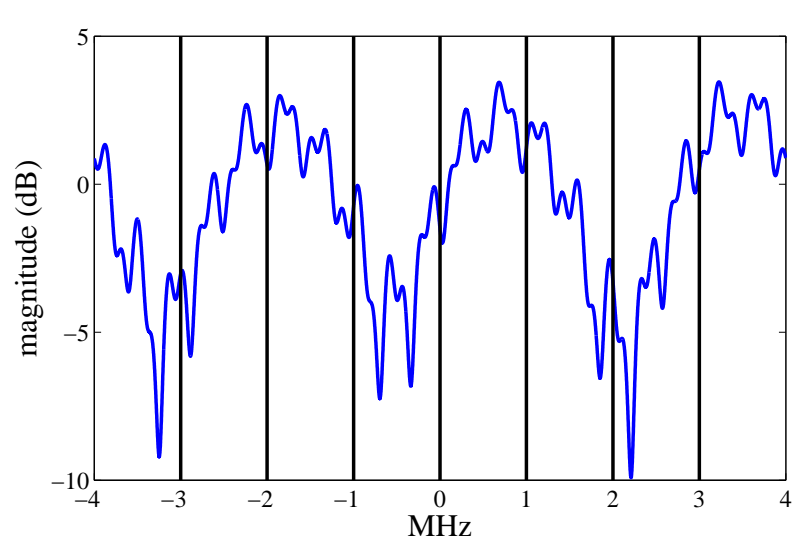

Fig. 1. Example of the baseband transfer function of a TU6 channel.

around $13.5 \mathrm{~dB}$. From this figure, we can see that the deep fading does not happen everywhere and only happens in the frequency band of $-4 \sim-3 \mathrm{MHz}$ and $2 \sim 3 \mathrm{MHz}$. By discarding this $2 \mathrm{MHz}$ sub band, the dynamic range of the channel is reduced to $10.7 \mathrm{~dB}$.

The current mobile TV standards do not support this idea, as all sub bands are considered equally important by the Forward Error Correction layer (FEC). Therefore, we propose a novel FEC layer based on fountain codes that does not have this disadvantage. In [4], MacKay describes the encoder of a fountain code as a metaphorical fountain that produces a stream of encoded packets. Anyone who wishes to receive the encoded file holds a bucket under the fountain and collects enough packets to recover the original data. It does not matter which packets are received, only a minimum amount of packets have to be received correctly [4]. In other words, fountain-encoded packets are independent with respect to each other.

To apply fountain codes in mobile TV, we divide a block of source bits into a set of packets, which are encoded by a fountain code. A fountain-encoded packet is transmitted over a sub band of the channel. Thus, multiple packets are transmitted simultaneously, using frequency division multiplexing. The receiver discards fountain-encoded packets which are transmitted over the sub band with deep fading. Correspondingly, the power consumption in ADCs decreases.

In this paper, we propose an approach based on resolution adaptive ADCs and fountain codes to reduce the power consumption in ADCs. The proposed method is an opportunistic error correction layer because it does not process all received 
packets but only processes surviving packets. This error correction layer is able to cope with discarding packets because fountain-encoded packets are independent of each other. Also, less power is consumed as the resolution of ADC is adapted to the minimum for each case, compared to using the fixedresolution ADC. Thus, it is a novel cross-layer approach which integrates the error correction into the physical layer of an OFDM system.

The outline of this paper is as follows. Fountain codes and a resolution adaptive ADC are applied to lower the power consumption in mobile TV receivers. First, fountain codes are discussed which is followed by the resolution adaptive ADC. In section IV, a description is given of the DVB-H system, which is a practical example of mobile TV. We compare the current DVB-H system with our proposed system in the simulation. Finally, the simulation results are analyzed. The paper ends with a discussion of the results.

\section{FOUNTAIN CODES}

In this paper, we use a kind of fountain codes, i.e. Luby Transform (LT) codes [5] in the proposed error correction layer. Other fountain codes (e.g. Raptor codes [6]) can also be applied.

Consider a block of size $K$ packets $s_{1}, s_{2}, \cdots, s_{K}$ to be encoded by a fountain code. A packet has $m$ bits and considered a unit. At each clock cycle, labeled by $n$, one fountainencoded packet is generated by selecting a set of source packets randomly and computing the bitwise sum (XOR) of these source packets [4]. The fountain codes can supply an unlimited number of encoded packets based on $s_{1}, s_{2}, \cdots, s_{K}$. In practical systems, only a fixed number of packet $N$ is generated.

At the receiver side, enough packets are required for successful decoding. The required number of received packets $N$ is slightly larger than the number of source packets $K$ and is defined as:

$$
N=K(1+\varepsilon)
$$

where $\varepsilon$ is the percentage of extra packets and is called the overhead.

After receiving $N$ packets, the receiver can recover the source packets by the message-passing algorithm [7] which has a linear decoding cost. We have shown that decoding the fountain codes using the message-passing algorithm combined with Gaussian elimination allows small block sizes, e.g. $K=500$, with small overhead $\varepsilon=3 \%$ in [8]. Small block sizes are needed to keep the decoding delay low, which is important in real-time applications such as mobile TV.

Fountain codes are designed for Erasure Channels. However, wireless channels are noisy channels, not erasure channels. In practical systems, fountain codes are used in combination with other error correction algorithms to convert the noisy channels into erasure channels, often Low-Density Parity-Check (LDPC) codes [7]. In this paper, LDPC codes are used together with a Cyclic Redundancy Check (CRC) to make the wireless channel behave like an erasure channel.
Our FEC encoding scheme is performed in the following order. First, a fountain-encoded packet is created. Then, the CRC is added. Finally, the packet is encoded by the LDPC code.

At the receiver, each fountain-encoded packet is first LDPC decoded if its energy is above or equal to a threshold (i.e. corresponding to BER $\leq 10^{-5}$ ). The received packet is discarded if its energy is below the threshold. If the LDPC decoding fails, the received packet is discarded as well. If the LDPC decoding succeeds, the CRC is used to identify any errors undetected by the LDPC codes. If the CRC decoder detects an error, the receiver assumes that the whole packet has been lost. Once the receiver gets $N$ surviving fountainencoded packets, it starts to recover the source data.

\section{RESOLUTION ADAPTIVE ADC}

In [9], the authors have designed an resolution adaptive ADC for IEEE 802.11a WLAN system and shown that adaptive resolution ADCs can save $38 \%$ power consumption in ADCs comparing with conventional high-resolution ADCs. For WLAN receivers, ADC power consumption can be almost $50 \%$ of the total baseband power consumption [1]. For a mobile TV receiver, we expect that similar values. An CMOS implementation of such an ADC is described in [3]. In this implementation, the power consumption scales linearly with the number of quantization levels.

\section{A. Minimum Number of Quantization Levels}

The mobile TV channel is often modeled as a time-varying frequency selective channel. The TU6 channel model is representative for the typical mobile reception with the Doppler frequency above $10 \mathrm{~Hz}$ [2].

In OFDM receivers, demodulation of the sub carriers is performed in the frequency domain. For that reason, it is not beforehand clear, how many ADC bits are necessary for proper decoding. In [9], the authors have derived an relation between the quantization noise in the time domain and the frequency domain for frequency selective channels. Here, we briefly explain it following [9].

We assume that the channel is noiseless and the channel of one OFDM symbol is constant, so the channel output at the $n^{\text {th }}$ moment $r_{n}$ is defined as:

$$
r_{n}=\sum_{l=0}^{L-1} h_{l} x_{n-l}
$$

where $L$ is the number of channel taps, $h_{l}$ the channel taps and $x$ the transmitted signal. We assume that the quantization noise is dominant, so other noise (e.g. thermal noise) is ignored in this paper. In [9], it has shown that $r_{n}$ is Gaussian-distributed with a zero mean and a variance of $\sum_{l}\left|h_{l}\right|^{2}$.

The ADC output $y_{n}$ is expressed by:

$$
y_{n}=\mathcal{Q}\left(r_{n}\right)=\sum_{l} h_{l} x_{n-l}+n_{n}
$$

where $n_{n}$ is the quantization noise in the time domain. Because the quantization noise $n_{n}$ depends on the signal $r_{n}$ and the 
probability density function of $r_{n}$ is symmetric to $0, n_{n}$ is uniform distributed with zero mean and a variance of $\frac{\Delta^{2}}{6}$, where $\Delta$ is the uniform quantization step [10].

After the OFDM demodulation, we have $Y_{k}$ as [11]:

$$
Y_{k}=H_{k} X_{k}+N_{k}
$$

where $N_{k}$ is the quantization noise in the frequency domain defined by [9]:

$$
N_{k}=\frac{1}{\sqrt{\mathrm{N}}} \sum_{n} n_{n} e^{-j \frac{2 \pi}{\mathrm{N}} n k}
$$

and $H_{k}$ is the fading over the $k^{t h}$ sub carrier defined by:

$$
H_{k}=\sum_{l} h_{l} e^{-j \frac{2 \pi}{N} l k}
$$

$N_{k}$ is a Gaussian-distributed random variable with zero mean and a variance of $\frac{\Delta^{2}}{6}$ according to the Central Limit Theorem [9]. Thus, for each sub carrier, the variance of the quantization noise is the same, but the Signal-to-(quantization)-Noise Ratio (SNR) is different due to different fading:

$$
\mathrm{SNR}_{k}=\frac{\left|H_{k}\right|^{2}}{\frac{\Delta^{2}}{6}}
$$

If the clipping is allowed, the number of quantization levels $N_{q}$ is determined by [10]:

$$
N_{q}=2\left\lceil\frac{\mathrm{C}}{\Delta}\right\rceil
$$

where $\mathrm{C}$ is equal to $3 \sigma_{r_{n}}$. Once the channel is fixed, $N_{q}$ is only determined by $\Delta$. In such case, $\Delta$ depends not only on the applied error correction codes in the system (i.e. SNR defined in Equation 7), but also on how the fountain-encoded packets are transmitted.

\section{B. Transmission Scheme}

In [9], the authors have shown that each fountain-encoded packet is transmitted over one sub carrier and the dynamic range of ADCs can be reduced by discarding the low-energy sub carriers for each channel realization. In the $802.11 \mathrm{a}$ WLAN system, the channel can be considered to be timeinvariant over a MAC frame. In [9], the transmission of each fountain-encoded packet is finished within a MAC frame. Hence, the channel over one fountain-encoded packet transmission can be considered as a time-invariant flat fading channel. However, this does not apply in the mobile TV system.

The mobile TV transmission system (e.g. DVB-H) should offer sufficient flexibility and scalability to allow the reception of the services at various speeds while optimizing transmission coverage [2]. In this paper, we focus on the $8 \mathrm{k}$ transmission mode which has a large area of the Single-Frequency Network (SFN) and maximum frequency efficiency [12]. Also, the 8k mode is more sensitive to the Doppler spread comparing to other transmission modes. In order to avoid the effects of the Doppler spread in adjusting resolution adaptive ADCs, we propose to transmit each fountain-encoded packet over a set

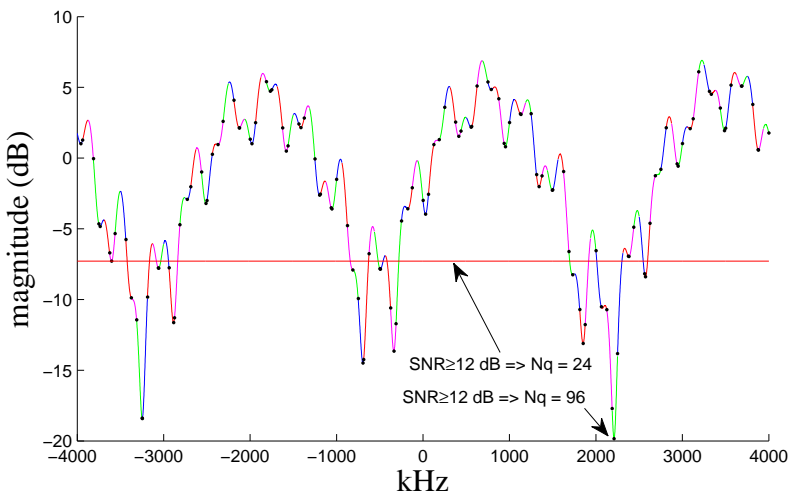

Fig. 2. An example of dividing TU6 channel into 128 sub bands. The black point is the sub carrier with the lowest energy every sub band, which defines the energy of its corresponding sub band.

of adjacent sub carriers. We denote such a set of sub carriers a sub band. In such case, the $8 \mathrm{k}$ sub carriers can be divided into a number of sub bands. Over each sub band, one fountainencoded packet is transmitted. The energy of each sub band is equal to the lowest energy of the sub carrier in its sub band.

Since each fountain-encoded packet is independent, it does not matter that we discard some packets which are transmitted over low-energy sub bands. For example, assume that the SNR of the high-energy sub band should be at least equal to $12 \mathrm{~dB}$ and fountain-encoded packets are transmitted over a wireless channel as shown in Figure 2. We discard low-energy sub bands and keep high-energy sub bands. We denote the number of low-energy sub bands as $N_{b}$. If we keep all sub bands (i.e. $N_{b}=0$ ) in Figure 2, the required number of quantization levels $N_{q}$ is 96 ; but if we discard 30 sub bands (i.e. $N_{b}=30$ ), the required $N_{q}$ can be reduced to 24 .

\section{Power Consumption}

The power consumption of the ADC is proportional to the number of quantization levels $N_{q}$ which is related to the Effective Number Of Bits (ENOB) by:

$$
N_{q}=2^{\mathrm{ENOB}}
$$

Hence, $N_{q}$ is a measurement of the power consumption $P$ :

$$
P=\sum_{i=0}^{M_{c}-1} \alpha_{i} N_{q_{i}} \mathcal{M}
$$

where $M_{c}$ is the number of OFDM symbols, $\alpha_{i}$ is the percentage of the $i$-th OFDM symbol where useful information is transmitted, $N_{q_{i}}$ is the number of quantization levels used in the $i$-th OFDM symbol, and $\mathcal{M}$ is the number of samples per OFDM symbol.

The power consumption in ADCs can be reduced by discarding low-energy sub bands. However, discarding transmitted packets over low-energy sub bands results in an increase of the total number of transmitted packets. Therefore, there is a tradeoff in power consumption between the number of lowenergy sub bands and the total number of transmitted packets. 


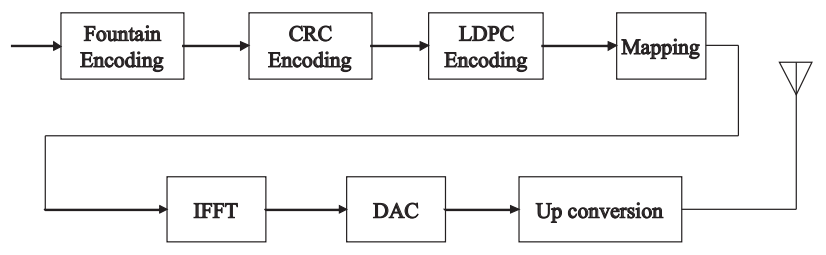

(a) Transmitter

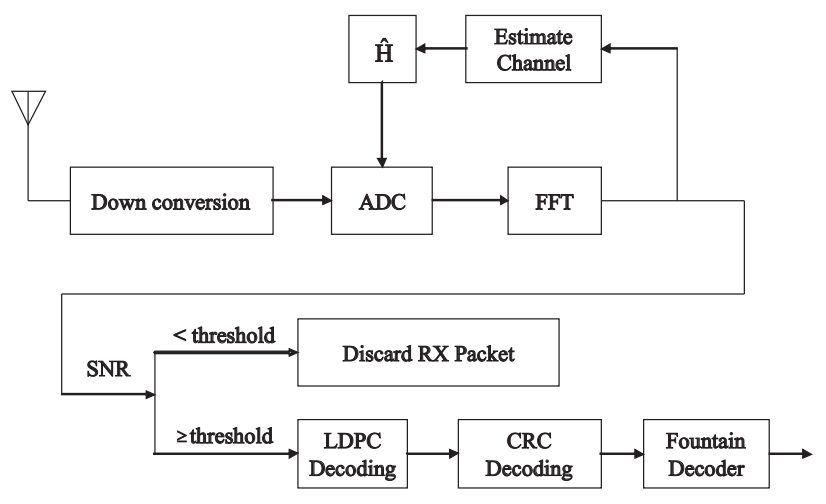

(b) Receiver

Fig. 3. Proposed DVB-H transmitter (top) and receiver (bottom).

So far, we design the quantization scheme for each OFDM symbol according to the current channel knowledge. However, in a practical mobile TV system (e.g. DVB-H), pilot symbols are only located in the scattered sub carriers, which means that the pilot data and user data are transmitted in the same OFDM symbol at the same time. In other words, it is impossible to adjust the resolution of ADCs to the minimum according to the current channel knowledge. Therefore, we have to use the channel knowledge of the previous OFDM symbol to adjust the resolution of ADCs for quantizing the current OFDM symbol, which will of course negatively affect the design of quantization scheme. We will discuss this influence in the next section.

\section{SYSTEM MODEL}

The opportunistic error correction layer is based on fountain codes and resolution adaptive ADCs which have explained in the above sections. This proposed cross layer can be applied in mobile TV systems. In this paper, the DVB-H system is taken as an example of mobile TV systems.

The FEC layer in the current DVB-H system is based on Reed Solomon (RS) codes and Rate Compatible Punctured Codes (RCPC). These codes have a good performance for random bit errors. Interleaver is employed to remove burst errors. Although this solution works well in practical systems, it is not optimal. First, because packets that have encountered a low-energy channel are still processed by the decoders. It will waste processing power. In addition, the error correction layer is based on worst case scenarios. This means that for most packets, the code rate and hence capacity can be increased. Furthermore, the resolution of the applied ADCs is fixed for DVB-H receivers, which is designed for worse case conditions. However, worse case conditions do not happen often.

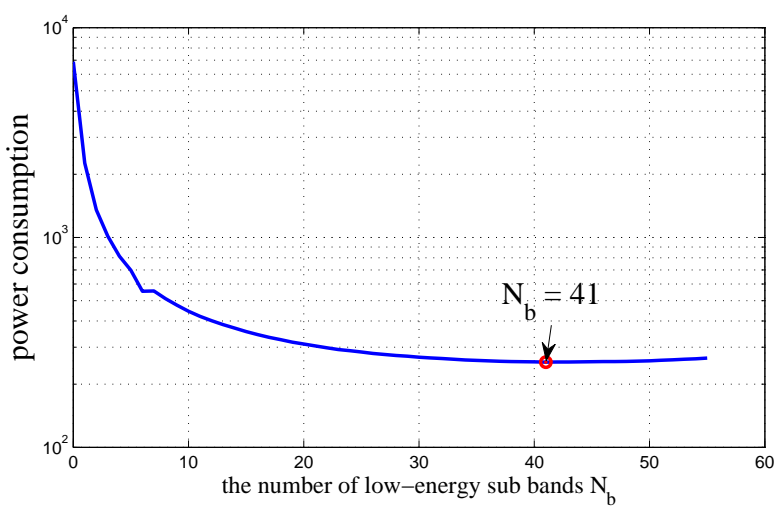

Fig. 4. The average power consumption versus the number of low-energy sub bands for receiving a burst of fountain-encoded packets. 64 sub carriers are grouped into a sub band and the total number of active sub bands is 106 .

In Figure 3, the proposed new error correction scheme is depicted to reduce the power consumption in ADCs. The key idea is to generate additional packets by the fountain encoder. First, the source packets are encoded by the fountain encoder. Then, a CRC checksum is added to each fountain-encoded packet and LDPC encoding is applied. On each sub band, a fountain-encoded packet is transmitted. Thus, multiple packets are transmitted simultaneously, using frequency division multiplexing.

At the receiver side, we assume that the synchronization and channel estimation are perfect. With the perfect channel knowledge, the resolution of the adaptive ADCs can be reduced to the minimum for each channel realization. If the SNR of the sub band is equal to or above the threshold, the received fountain-encoded packet will go through LDPC decoding, otherwise it will be discarded. This means that the receiver is allowed to discard low-energy sub bands (i.e. packets) to lower the dynamic range of the ADC and hence the power consumption. After the LDPC decoding, the CRC checksum is used to discard the erroneous packets. As only packets with a high SNR are processed by the receiver, this will not happen often. When the receiver collects enough fountain-encoded packets, it starts to recover the source data.

In Figure 4, the relation is depicted between the average power consumption (dynamic range) of ADCs and the number of low-energy sub bands $N_{b}$, under the condition that the resolution of ADCs is adjusted according to the current channel knowledge. In each case, 1000 fountain-encoded packets are received and the low-energy sub bands are discarded. The number of the transmitted fountain-encoded packets is defined as $1000 \times \frac{N_{a}}{N_{a}-N_{b}}$, where $N_{a}$ is the total number of active sub bands. When 41 sub bands are discarded, the minimum power consumption is achieved.

As mentioned in Section III, it is impossible to adjust the resolution of $\mathrm{ADCs}$ according to the current channel knowledge. In order to apply the resolution adaptive ADCs in the DVB-H receiver, we propose to use the channel knowledge of the previous OFDM symbol to adjust the resolution of 


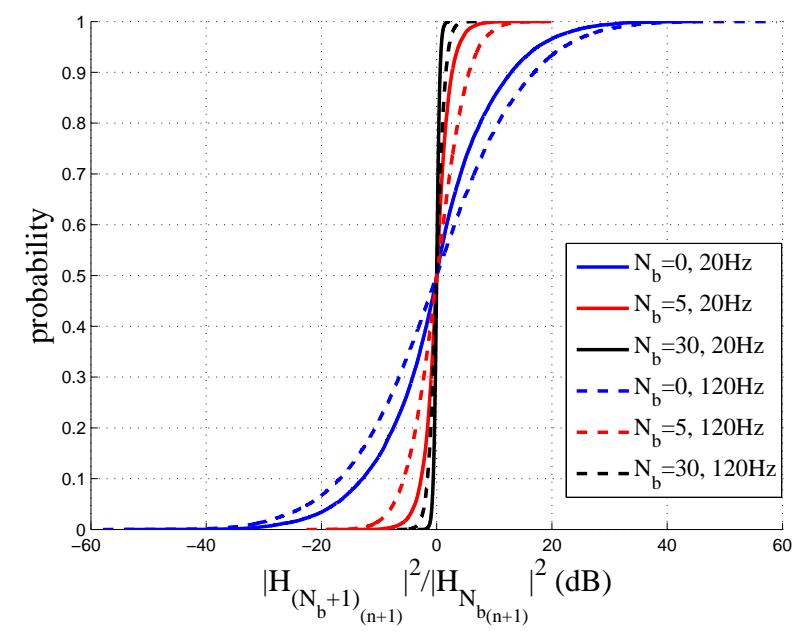

Fig. 5. The probability of $\frac{\left|H_{\left(N_{b}+1\right)}(n+1)\right|}{\left|H_{\left(N_{b}+1\right)_{n} \mid}\right|}$ for different $N_{b}$ and different Doppler shift. Those probability curves are based on $2 \times 10^{4}$ TU6 channels with $20 \mathrm{~Hz}$ and $120 \mathrm{~Hz}$ Doppler shift.

ADCs for the current OFDM symbol. In such case, we still achieve the lowest power consumption when we discard $41 \mathrm{sub}$ bands. However, the actual number of lost sub bands might not be as same as we expected and this needs more analysis.

We assume that the channel is perfectly estimated and $N_{b}$ sub bands are lost. We denote each sub band of the $n^{\text {th }}$ OFDM symbol by $H_{k_{n}}$ and we sort the sub band by the energy, so we have:

$$
\left|H_{(k+1)_{n}}\right|>\left|H_{k_{n}}\right|
$$

Suppose that the fountain-encoded packet can be decoded correctly when SNR $\geq 12 \mathrm{~dB}$. We design the quantization scheme for the $(n+1)^{t h}$ OFDM symbol by making the $\left(N_{b}+1\right)^{t h}$ sub band of the $n^{t h}$ OFDM symbol have a SNR of $12 \mathrm{~dB}$, so we have:

$$
\Delta=\sqrt{6 \frac{\left|H_{\left(N_{b}+1\right)}\right|^{2}}{10^{1.2}}}
$$

The SNR of the $\left(N_{b}+1\right)^{t h}$ sub band in the $(n+1)^{t h}$ OFDM symbol is defined by:

$$
\begin{aligned}
\operatorname{SNR}_{\left(N_{b}+1\right)_{(n+1)}} & =\frac{\left|H_{\left(N_{b}+1\right)_{(n+1)}}\right|^{2}}{\frac{\Delta^{2}}{6}} \\
& =12+20 \log _{10} \frac{\left|H_{\left(N_{b}+1\right)_{(n+1)}}\right|}{\left|H_{\left(N_{b}+1\right)_{n}}\right|}
\end{aligned}
$$

If $\left|H_{\left(N_{b}+1\right)_{(n+1)}}\right| \geq\left|H_{\left(N_{b}+1\right)_{n}}\right|, \operatorname{SNR}_{\left(N_{b}+1\right)_{(n+1)}} \geq 12 \mathrm{~dB}$; otherwise $\operatorname{SNR}_{\left(N_{b}+1\right)_{(n+1)}}<12 \mathrm{~dB}$. The difference between $\left|H_{\left(N_{b}+1\right)_{(n+1)}}\right|$ and $\left|H_{\left(N_{b}+1\right)_{n}}\right|$ determines whether the actual number of lost sub bands will be more than or less than or equal to $N_{b}$ sub bands.

$\frac{\left|H_{\left(N_{b}+1\right)(n+1)}\right|}{\left|H_{\left(N_{b}+1\right)_{n}}\right|}$ depends on the predefined number of lowenergy sub bands $N_{b}$ and the Doppler spread, as shown in Figure 5. From this figure, we can see that the gap is inversely proportional to $N_{b}$ but proportional to the Doppler spread. For the case of $N_{b}=0$, the range of $\frac{\mid H_{\left(N_{b}+1\right)_{(n+1)} \mid}}{\mid H_{\left(N_{b}+1\right)_{n} \mid}}$ is within \pm 27 $\mathrm{dB}$ at the probability of $95 \%$ for $120 \mathrm{~Hz}$ Doppler spread and at $97.5 \%$ for $20 \mathrm{~Hz}$ Doppler spread; but when $N_{b}=30$, the range can be reduced to $\pm 2.7 \mathrm{~dB}$ at $95 \%$ for $120 \mathrm{~Hz}$ and at $98 \%$ for $20 \mathrm{~Hz}$ Doppler spread. So, it is beneficial to discard more sub bands.

Due to the lack of a feedback channel, there is no chance to recover the fountain source packets if we lose more sub bands than we anticipated. To make sure that receivers get enough fountain-encoded packets, we can increase the designed resolution of ADC by a certain number of bits to compensate for the gap between $\left|H_{\left(N_{b}+1\right)_{(n+1)}}\right|$ and $\left|H_{\left(N_{b}+1\right)_{n}}\right|$. In such case, we should give 4.5 bits extra for $N_{b}=0$ and only 0.45 bit extra for $N_{b}=30$ to recover the source packets at least with the probability of $97.5 \%$.

\section{PERFORMANCE ANALYSIS}

In this section, we analyze the performance of our proposed opportunistic error correction layer. In the simulation, we transmit 1000 blocks of source packets to 1000 receivers over a TU6 channel with $120 \mathrm{~Hz}$ Doppler shift. Each block consists of 500 source packets with a length of 168 bits.

We compare two scenarios in the simulation. The first scenario, Scenario I, is a conventional DVB-H system with 16-QAM modulation and code rate $2 / 3 \times 3 / 4=1 / 2$ [12]. We allow that $1 \%^{2}$ receivers have packet loss and the other ones will receive the packets correctly. Moreover, we assume that the conventional fixed-resolution ADCs are used.

In Scenario II, we apply the new opportunistic error correction layer, which has the same effective throughput as Scenario I. Each burst is encoded by a LT code (with parameters $c=0.03, \sigma=0.3$ ) and decoded by the message-passing algorithm and Gaussian elimination together. From [8], we know that $3 \%$ overhead is required to recover the source packets successfully. To each fountain-encoded packet, a 7-bit CRC is added then the $(255,175)$ LDPC encoder is applied. Under the condition of the same effective throughput, we can lose 23 sub bands where the power consumption is closed to the minimum case with 41 lost sub bands as shown in Figure 4. For the resolution adaptive ADC, we use the perfect channel knowledge of the previous OFDM symbol to adjust the resolution of ADCs for the current OFDM symbol. In order to achieve the expected number of fountain-encoded packets (i.e. $N=515$ ) with the probability of at least $99 \%$, we will give some extra bits (i.e. $4 \mathrm{~dB}$ extra SNR).

In our simulations, we have used the parameters in Table I to compare the conventional and the proposed system. The "SNR in frequency domain" is the minimal SNR for all sub bands in Scenario I and the minimal SNR for all high-energy sub bands in Scenario II.

Figure 6 shows the average power consumption in ADCs for the transmission of each block. We normalize the power consumption of Scenario I to $100 \%$. From this figure, we can

\footnotetext{
${ }^{2} \mathrm{~A}$ common value used in coverage tools
} 


\begin{tabular}{r|c|c} 
& Scenario I & Scenario II \\
\hline ADC & uniform & res. adapt. \\
FEC & RS+RCPC & LDPC + Fount. codes \\
Code rate & 0.5 & 0.64 \\
Modulation & $16-\mathrm{QAM}$ & $16-\mathrm{QAM}$ \\
$N_{c}$ & 6784 & 6784 \\
$N_{s}$ & 106 & 106 \\
$N_{b}$ & $9.0 \mathrm{~dB}$ & $16.0 \mathrm{~dB}$ \\
SNR in freq. domain & 0 & 23
\end{tabular}

TABLE I

SYSTEM SETUP COMPARISON FOR TWO SCENARIOS ( $N_{c}$ - THE NUMBER OF DATA CARRIERS, $N_{s}$ - THE NUMBER OF SUB BANDS AND $N_{b}$ - THE NUMBER OF LOW-ENERGY SUB BANDS)

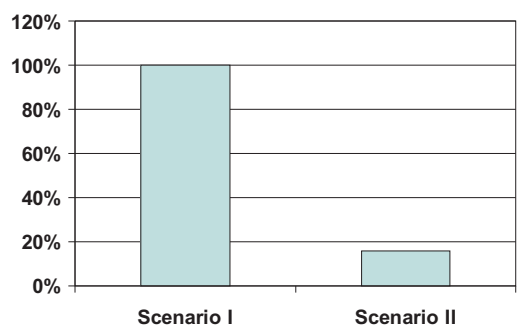

Fig. 6. Power consumption comparison between two Scenarios. The power consumption in ADCs of Scenario I is normalized to $100 \%$.

see that the power consumed in Scenario II is reduced to $16 \%$ in comparison with Scenario I.

In order to let the number of lost sub bands not be more than we expected (i.e. $N_{b}=23$ ), we give $4 \mathrm{~dB}$ extra SNR to adjust the resolution of ADCs in Scenario II. During the transmission of 1000 bursts, each receiver loses on average 1.1 sub bands and at maximum 13 sub bands every OFDM symbol, which means every receiver can recover the source packets correctly. So our system has a higher QoS at a lower power consumption compared to the conventional system where typically $1 \%$ of the receivers can not decode the mobile TV stream.

\section{CONCLUSIONS}

In this paper, we propose a novel cross-layer scheme which integrates the error correction into the physical layer. This new opportunistic error correction layer is especially designed for mobile TV (e.g. DVB-H) and broadcasting applications. It is based on fountain codes and resolution adaptive ADCs. Each fountain-encoded packet is transmitted over a sub band which consists of a set of sub carriers. By discarding fountainencoded packets that have been transmitted over low-energy sub bands, the dynamic range of ADCs can be reduced. Correspondingly, the power consumption in ADCs can be decreased. The new cross-layer method results in up to $84 \%$ lower power consumption of the ADCs compared to the current standard. Moreover, it has higher coverage than the current standard.

\section{ACKNOWLEDGEMENTS}

The authors thank Niels A. Moseley for the useful comments and discussions. Also, the authors acknowledge the
Dutch Ministry of Economic Affairs under the IOP Generic Communication - Senter Novem Program for the financial support.

\section{REFERENCES}

[1] J. Thomson, et al., "An Integrated 802.11a Baseband and MAC Processor," Solid-State Circuits Conference, 2002. Digest of Technical Papers. ISSCC. 2002 IEEE International, vol. 1, pp. 126-451 vol.1, 2002.

[2] G. Faria, J. A. Henriksson, E. Stare and P. Talmola, "DVB-H: Digital Broadcast Services to Handheld Devices," in Proceedings of the IEEE, vol. 94, no. 1, Jan. 2006

[3] R. van de Plassche, CMOS Integrated Analog-to-Digital and Digital-toAnalog Converters. Kluwer Academic Publishers, 2003.

[4] D. J. C. MacKay, "Fountain Codes," IEE Communications, vol. 152, no. 6, pp. 1062-1068, 2005.

[5] M. Luby, "LT Codes," Proceedings of the 43rd Annual IEEE Symposium on Foundations of Computer Science, pp. 271-282, 2002.

[6] A. Shokrollahi, "Raptor Codes," IEEE Transaction on Information Theory, vol. 52, 2006.

[7] D.J.C. MacKay, Information Theory, Interference, and Learning Algorithms. Cambridge, UK: Cambridge University Press, 2003.

[8] X. Shao, R. Schiphorst and C. H. Slump, "An Opportunistic Error Correction Layer for OFDM Systems," EURASIP Journal on Wireless Communications and Networking, submitted for publication.

[9] — - "Opportunistic Error Correction for WLAN Applications," in Proceedings of the 4th IEEE International Conference on Wireless Communications, Networking and Mobile Computing, Da Lian, China, Oct. 2008.

[10] X. Shao and C. H. Slump, "Quantization Effects in OFDM Systems," in Proceedings of 29th Symposisum on Information Theory in the Benelux, Leuven, May 2008.

[11] D. Tse and P. Viswanath, Fundamentals of Wireless Communication. New York, NY, USA: Cambridge University Press, 2005.

[12] Digital Video Broadcasting (DVB); Framing Structure, Channel Coding and Modulation for Digital Terrestrial Television, European Telecommunications Standards Institute Std. ETSI EN 300744 V1.5.1, 2004 